Tôhoku Math. Journ.

39 (1987), 17-25.

\title{
BEHAVIOR OF SOLUTIONS FOR THE LINEARIZED \\ NAVIER-STOKES EQUATIONS WITH \\ VANISHING VISCOSITY
}

\author{
KIYOKAZU NAKAGAWA
}

(Received January 17, 1986)

1. Introduction. Let $\Omega$ be a bounded domain in $E_{3}$ with smooth boundary $\partial \Omega$ and let $T>0$ be fixed. For a vector function $\boldsymbol{v}_{\nu}=$ $\left(v_{\nu, 1}, v_{\nu, 2}, v_{\nu, 3}\right)$ and a scalar function $p_{\nu}$ representing the velocity of the fluid and the pressure we consider in $\Omega \times[0, T]$ an initial-boundary value problem for the linearized Navier-Stokes equation:

$$
\left\{\begin{array}{l}
D_{t} \boldsymbol{v}_{\nu}-\nu \Delta \boldsymbol{v}_{\nu}+\nabla p_{\nu}=\boldsymbol{f}, \\
\operatorname{div} \boldsymbol{v}_{\nu}=0, \\
\left.\boldsymbol{v}_{\nu}\right|_{t=0}=\boldsymbol{a},\left.\quad \boldsymbol{v}\right|_{\partial \Omega}=\mathbf{0},
\end{array}\right.
$$

where $\boldsymbol{a}(x), \boldsymbol{f}(x, t)$ are given vector functions and $\nu$ is the so-called viscosity coefficient.

The existence and uniqueness results for (1.1) are now well known (see, for instance, [1]); whereas the behavior of the solutions, as the viscosity coefficient $\nu$ tends to zero, is not yet fully understood, and we wish to study such a question in this note.

We first introduce our notation. Let $L^{2}(\Omega)$ be the Hilbert space of square integrable real functions on $\Omega$ and let $W^{k, 2}(\Omega)$ be the Sobolev space of functions with square integrable derivatives up to the order $k$ in $L^{2}(\Omega)$. For vector valued functions $\boldsymbol{v}=\left(v_{1}, v_{2}, v_{3}\right)$ the corresponding spaces are denoted by $\boldsymbol{L}^{2}(\Omega)$ and $\boldsymbol{W}^{k, 2}(\Omega)$. The norms will be denoted by \|\|$_{L^{2}(\Omega)}$, \|\|$_{W^{k, 2}(\Omega)}$ etc. Let $C_{0}^{\infty}(\Omega)=\left\{\boldsymbol{v}=\left(v_{1}, v_{2}, v_{3}\right) ; v_{i} \in C^{\infty}(\Omega), \operatorname{supp}\left(v_{i}\right) \subset \Omega, i=\right.$ $1,2,3\}$ and $\boldsymbol{C}_{0, o}^{\infty}(\Omega)=\left\{\boldsymbol{v} \in \boldsymbol{C}_{0}^{\infty}(\Omega)\right.$; $\left.\operatorname{div} \boldsymbol{v}=0\right\}$. We define $\boldsymbol{L}_{\sigma}^{2}(\Omega)$ and $\boldsymbol{H}(\Omega)$ as the closures of $\boldsymbol{C}_{0, \sigma}^{\infty}(\Omega)$ in $\boldsymbol{L}^{2}(\Omega)$ and $\boldsymbol{W}^{1,2}(\Omega)$. The orthogonal projection from $\boldsymbol{L}^{2}(\Omega)\left(\operatorname{resp} . \boldsymbol{L}^{2}(\Omega \times(0, T))=L^{2}\left(0, T ; \boldsymbol{L}^{2}(\Omega)\right)\right)$ onto $\boldsymbol{L}_{\sigma}^{2}(\Omega)\left(\operatorname{resp} . L^{2}\left(0, T ; \boldsymbol{L}_{\sigma}^{2}(\Omega)\right)\right)$ will be denoted by $\boldsymbol{P}_{\sigma}$.

We now assume in (1.1) that $\boldsymbol{a} \in \boldsymbol{H}(\Omega)$ and $\boldsymbol{f} \in \boldsymbol{L}^{2}(\Omega \times(0, T))$. Then as will be seen in the next section, we have the solution $\left(\boldsymbol{v}_{\nu}, p_{\nu}\right)$, which as $\nu \rightarrow 0$ converges weakly in the Hilbert space $\boldsymbol{L}^{2}(\Omega \times(0, T)) \times L^{2}(\Omega \times(0, T))$ to the solution $\left(\boldsymbol{v}_{0}, p_{0}\right)$, given by 


$$
\left\{\begin{array}{l}
\boldsymbol{v}_{0}(x, t)=\boldsymbol{a}(x)+\int_{0}^{t} \boldsymbol{P}_{o} \boldsymbol{f}(x, \tau) d \tau, \\
\nabla p_{0}(x, t)=\left(\boldsymbol{I}-\boldsymbol{P}_{\sigma}\right) \boldsymbol{f}(x, t),
\end{array}\right.
$$

of the equations:

$$
\left\{\begin{array}{l}
D_{t} \boldsymbol{v}_{0}+\nabla p_{0}=\boldsymbol{f}, \\
\operatorname{div} \boldsymbol{v}_{0}=0, \\
\left.\boldsymbol{v}_{0}\right|_{t=0}=\boldsymbol{a},
\end{array}\right.
$$

obtained from (1.1) by setting $\nu=0$ and eliminating the physically required boundary condition due to the viscosity of the fluid.

Our purpose here is to show that the solution $\left(\boldsymbol{v}_{\nu}, p_{\nu}\right)$ actually enjoys a better convergence property in a domain strictly away from the boundary.

We introduce a partial fractional integration

$$
I^{\alpha} p_{\nu}(x, t)=(1 / \Gamma(\alpha)) \int_{0}^{t}(t-\tau)^{\alpha-1} p_{\nu}(x, \tau) d \tau \quad(\alpha>0)
$$

with respect to the time variable $t$, where $\Gamma(\alpha)$ is the Gamma function; also, in this note we always suppose that the pressure function $p_{\nu}(x, t)$ is subjected to the condition

$$
\int_{\Omega} p_{\nu}(x, t) d x=0
$$

for almost all $t$ in $[0, T]$, so that $p_{\nu}(x, t)$ is essentially uniquely determined from $\nabla p_{\nu}(x, t)$.

Our result is now as follows.

THEOREM 1.1. If $\boldsymbol{a} \in \boldsymbol{H}(\Omega), \boldsymbol{f} \in \boldsymbol{L}^{2}(\Omega \times(0, T))$ and if $V$ is an arbitrary compact subdomain of $\Omega$, then as $\nu \rightarrow 0$

(1) $\boldsymbol{v}_{\nu}$ converges strongly to $\boldsymbol{v}_{0}$ in $\boldsymbol{L}^{2}(V \times(0, T))$,

(2) for any $\alpha>0 I^{\alpha} p_{\nu}$ converges strongly to $I^{\alpha} p_{0}$ in $L^{2}(V \times(0, T))$.

This result can be stated in a stronger form by assuming the data $\boldsymbol{a}, \boldsymbol{f}$ sufficiently smooth. Such a result will be presented in Section 5 .

2. Weak convergence of solutions for vanishing viscosity. According to [1], we have the following existence theorem:

THeOREM 2.1. Assume that $\boldsymbol{a} \in \boldsymbol{H}(\Omega), \boldsymbol{f} \in \boldsymbol{L}^{2}(\Omega \times(0, T))$ and (1.5) for $p_{\nu}$. Then, there exists a unique solution $\left(\boldsymbol{v}_{\nu}, p_{\nu}\right)$ of (1.1) such that $\boldsymbol{v}_{\nu} \in L^{2}\left(0, T ; W^{2,2}(\Omega)\right), D_{t} \boldsymbol{v}_{\nu} \in L^{2}\left(0, T ; L_{o}^{2}(\Omega)\right), p_{\nu} \in L^{2}\left(0, T ; W^{1,2}(\Omega)\right)$, and moreover that $\boldsymbol{v}_{\nu}(t) \in C([0, T] ; \boldsymbol{H}(\Omega))$, i.e., $\boldsymbol{v}_{\nu}(t)$ is continuous on $[0, T]$ with values in $\boldsymbol{H}(\Omega)$. For this solution we have the following estimates: 


$$
\begin{aligned}
& \left\|D_{t} \boldsymbol{v}_{\nu}\right\|_{\boldsymbol{L}^{2}(\Omega \times(0, T))}+\nu^{1 / 2} \sum_{j=1}^{3}\left\|D_{j} \boldsymbol{v}_{\nu}\right\|_{\boldsymbol{L}^{2}(\Omega \times(0, T))} \\
& +\left\|\boldsymbol{v}_{\nu}\right\|_{\boldsymbol{L}^{2}(\Omega \times(0, T))} \leqq C\left(\|\boldsymbol{f}\|_{\boldsymbol{L}^{2}(\Omega \times(0, T))}+\sum_{j=1}^{3}\left\|D_{j} \boldsymbol{a}\right\|_{\boldsymbol{L}^{2}(\Omega)}\right), \\
& \left\|\boldsymbol{v}_{\nu}(t)\right\|_{\boldsymbol{L}^{2}(\Omega)} \leqq C\left(\|\boldsymbol{f}\|_{\boldsymbol{L}^{2}(\Omega \times(0, T))}+\|\boldsymbol{a}\|_{\boldsymbol{L}^{2}(\Omega)}\right)
\end{aligned}
$$

for $t \in[0, T]$ where $D_{j}=\partial / \partial x_{j}$ and $C$ is a constant independent of $\nu$.

Now, concerning the weak convergence, we have

THEOREM 2.2. Let the assumption on $\boldsymbol{a}, \boldsymbol{f}, p_{\nu}$ be the same as in Theorem 2.1, and let $\left(\boldsymbol{v}_{0}, p_{0}\right)$ be the solution of (1.3) given by (1.2). Then,

(1) the vector $\boldsymbol{v}_{\nu}$ (resp. $\left.D_{t} \boldsymbol{v}_{\nu}, p_{\nu}\right)$ converges weakly to $\boldsymbol{v}_{0}\left(\operatorname{resp} . D_{t} \boldsymbol{v}_{0}, p_{0}\right)$ in $\boldsymbol{L}^{2}(\Omega \times(0, T))$ as $\nu \rightarrow 0$.

(2) For every $t \in[0, T]$ the vector $\boldsymbol{v}_{\perp}(t)$ converges weakly to $\boldsymbol{v}_{0}(t)$ in $\boldsymbol{L}_{\sigma}^{2}(\Omega)$ as $\nu \rightarrow 0$.

Proof. Let $\Phi$ be an arbitrary continuous function on $[0, T]$ with values in $C_{0, \sigma}^{\infty}(\Omega)$. We then have the identity

$$
\int_{0}^{T} \int_{\Omega} D_{t} \boldsymbol{v}_{\nu} \cdot \boldsymbol{\Phi} d x d t+\nu \sum_{j=1}^{3} \int_{0}^{T} \int_{\Omega} D_{j} \boldsymbol{v}_{\nu} \cdot D_{j} \boldsymbol{\Phi} d x d t=\int_{0}^{T} \int_{\Omega} \boldsymbol{P}_{o} \boldsymbol{f} \cdot \boldsymbol{\Phi} d x d t
$$

where the dot - means the pointwise scalar product of two vectors. From the estimate (2.1), follows therefore

$$
\lim _{\nu \rightarrow 0} \int_{0}^{T} \int_{\Omega} D_{t} \boldsymbol{v}_{\nu} \cdot \boldsymbol{\Phi} d x d t=\int_{0}^{T} \int_{\Omega} \boldsymbol{P}_{o} \boldsymbol{f} \cdot \boldsymbol{\Phi} d x d t .
$$

By continuity, this relation holds for every $\boldsymbol{\Phi} \in L^{2}\left(0, T ; \boldsymbol{L}_{\sigma}^{2}(\Omega)\right)$; also for every $\boldsymbol{\Phi} \in L^{2}\left(0, T ; \boldsymbol{L}^{2}(\Omega)\right)$, since both $D_{t} \boldsymbol{v}_{\nu}$ and $\boldsymbol{P}_{o} \boldsymbol{f}$ belong to $L^{2}\left(0, T ; \boldsymbol{L}_{\sigma}^{2}(\Omega)\right)$. Hence $D_{t} \boldsymbol{v}_{\nu}$ converges weakly to $D_{t} \boldsymbol{v}_{0}=\boldsymbol{P}_{o} \boldsymbol{f}$ in $\boldsymbol{L}^{2}(\Omega \times(0, T))$ as $\nu \rightarrow 0$.

Let $\boldsymbol{\Psi} \in \boldsymbol{L}^{2}(\Omega)$. Then

$$
\int_{\Omega} \boldsymbol{v}_{\nu}(t) \cdot \Psi d x=\int_{\Omega} \boldsymbol{a} \cdot \Psi d x+\int_{0}^{t} \int_{\Omega} D_{\tau} \boldsymbol{v}_{\nu}(\tau) \cdot \Psi d x d \tau
$$

for every $t \in[0, T]$. From the result just obtained there now follows the weak convergence of $\boldsymbol{v}_{\nu}(t)$ to $\boldsymbol{v}_{0}(t)=\boldsymbol{a}+\int_{0}^{t} \boldsymbol{P}_{\sigma} \boldsymbol{f}(\tau) d \tau$ in $\boldsymbol{L}^{2}(\Omega)$, which proves (2). The weak convergence of $\boldsymbol{v}_{\nu}$ to $\boldsymbol{v}_{0}$ in $\boldsymbol{L}^{2}(\Omega \times(0, T))$ is also clear, since $\boldsymbol{v}_{\nu}(t) \in C([0, T] ; \boldsymbol{H}(\Omega))$.

As regards the pressure $p_{\nu}$, we consider the relation

$$
\int_{0}^{T} \int_{\Omega} D_{t} \boldsymbol{v}_{\nu} \cdot \boldsymbol{\Phi} d x d t+\nu \sum_{j=1}^{3} \int_{0}^{T} \int_{\Omega} D_{j} \boldsymbol{v}_{\nu} \cdot D_{j} \boldsymbol{\Phi} d x d t+\int_{0}^{T} \int_{\Omega} \nabla p_{\nu} \cdot \boldsymbol{\Phi} d x d t=\int_{0}^{T} \int_{\Omega} \boldsymbol{f} \cdot \boldsymbol{\Phi} d x d t
$$

which holds for every $\Phi \in L^{2}\left(0, T ; \boldsymbol{W}_{0}^{1,2}(\Omega)\right)$. Here $\boldsymbol{W}_{0}^{1,2}(\Omega)$ is the closure of $\boldsymbol{C}_{0}^{\infty}(\Omega)$ in $\boldsymbol{W}^{1,2}(\Omega)$. We now let $\nu \rightarrow 0$ in this relation. Then since $D_{t} \boldsymbol{v}_{\nu}$ converges weakly to $D_{t} \boldsymbol{v}_{0}=\boldsymbol{P}_{\sigma} \boldsymbol{f}$ and the second term vanishes in the limit, we obtain 


$$
\lim _{\nu \rightarrow 0} \int_{0}^{T} \int_{\Omega} \nabla p_{\nu} \cdot \boldsymbol{\Phi} d x d t=\int_{0}^{T} \int_{\Omega}\left(\boldsymbol{I}-\boldsymbol{P}_{\sigma}\right) \boldsymbol{f} \cdot \boldsymbol{\Phi} d x d t=\int_{0}^{T} \int_{\Omega} \nabla p_{0} \cdot \boldsymbol{\Phi} d x d t
$$

in view of (1.2), or equivalently

$$
\lim _{\nu \rightarrow 0} \int_{0}^{T} \int_{\Omega} p_{\nu} \chi d x d t=\int_{0}^{T} \int_{\Omega} p_{0} \chi d x d t
$$

with $\chi=\operatorname{div} \Phi$, since $\Phi(x, t)$ vanishes on the boundary $\partial \Omega$. Now let $D=\left\{\chi \in L^{2}(\Omega \times(0, T)) ; \chi=\operatorname{div} \Phi, \Phi \in L^{2}\left(0, T ; W_{0}^{1,2}(\Omega)\right)\right\}$ and let $D^{\perp}$ denote the orthogonal complement of $D$ in $L^{2}(\Omega \times(0, T))$. Then $L^{2}(\Omega \times(0, T))=$ $\bar{D} \oplus D^{\perp}, \bar{D}$ being the closure of $D$, and we easily infer that $D^{\perp}$ contains only the functions independent of the variable $x$. By our assumption (1.5), the above relation therefore holds for every element in $L^{2}(\Omega \times(0, T))$, and the proof of Theorem 2.2 is thus complete.

3. Solonnikov's integral representation. Let $\Omega_{\delta}=\left\{x \in \Omega\right.$; $\inf _{y \in \partial \Omega} \mid x-$ $y \mid>\delta\}$ and let there be given a compact subset $V$ of $\Omega$. Taking $\delta$ sufficiently small we shall suppose that $V$ is in $\Omega_{4 \delta}$. Let $\zeta(x)$ be a $C^{\infty}$ function which is equal to unity on $\Omega_{2 \delta}$ and vanishes identically outside $\Omega_{\delta}$. We put $\boldsymbol{w}_{\nu}=\zeta \boldsymbol{v}_{\nu}, q_{\nu}=\zeta p_{\nu}$, and regard these as functions on $E_{3} \times[0, T]$ with supports in $\bar{\Omega}_{\delta} \times[0, T]$. Now multiplying (1.1) by $\zeta$, we obtain a system of equations in $E_{3} \times[0, T]$ :

$$
\left\{\begin{array}{l}
D_{t} \boldsymbol{w}_{\nu}-\nu \Delta \boldsymbol{w}_{\nu}+\nabla q_{\nu}=\zeta \boldsymbol{f}+\boldsymbol{g}_{\nu}, \\
\operatorname{div} \boldsymbol{w}_{\nu}=\nabla \zeta \cdot \boldsymbol{v}_{\nu}, \\
\left.\boldsymbol{w}_{\nu}\right|_{t=0}=\zeta \boldsymbol{a},
\end{array}\right.
$$

where $\boldsymbol{g}_{\nu}$ denotes the vector $-2 \nu \sum_{j=1}^{3} D_{j} \zeta D_{j} \boldsymbol{v}_{\nu}-\nu \Delta \zeta \boldsymbol{v}_{\nu}+p_{\nu} \nabla \zeta$. Thus, we may think of $\left(\boldsymbol{w}_{\nu}, q_{\nu}\right)$ as the solution of this Cauchy problem. Let

$$
\left\{\begin{array}{l}
K(x)=-1 / 4 \pi|x|, \\
\Gamma_{\nu}(x, t)= \begin{cases}(4 \pi \nu t)^{-3 / 2} \exp \left(-|x|^{2} / 4 \nu t\right) & \text { for } t>0, \\
0 & \text { for } t<0,\end{cases} \\
T_{\nu}(x, t)=\left\{T_{\nu i j}(x, t)\right\}_{1 \leqq i, j \leqq 3}=\Gamma_{\nu}(x, t) I-\operatorname{Hess}\left(\Gamma_{\nu}{ }_{x} K\right)(x, t),
\end{array}\right.
$$

where $I$ is the $3 \times 3$ unit matrix and Hess $\left(\Gamma_{\nu} *_{x} K\right)(x, t)=\left\{D_{i} D_{j} \int_{E_{3}} \Gamma_{\nu}(x-y, t)\right.$. $K(y) d y\}_{1 \leqq i, j \leqq 3}$ with $*_{x}$ denoting the convolution with respect to $x$-variables. In the following we also use symbol * to mean the convolution in $(x, t)$ variables. Note here that $K(x), \Gamma_{\nu}(x, t)$ are the so-called fundamental solutions of the Laplacian $\Delta$ and the heat equation $D_{t}-\nu \Delta ; T_{\nu}$ is usually called Oseen's tensor.

Owing to Solonnikov [3], we may now represent the solution of the Cauchy problem (3.1) as 


$$
\left\{\begin{array}{l}
\boldsymbol{w}_{\nu}(x, t)=\nabla s_{\nu}(x, t)+\Gamma_{\nu} *_{x}(\zeta \boldsymbol{a}+\boldsymbol{b})(x, t)+T_{\nu} *\left(\zeta \boldsymbol{f}+\boldsymbol{g}_{\nu}\right)(x, t), \\
q_{\nu}(x, t)=\operatorname{div}\left(K *_{x}\left(\zeta \boldsymbol{f}+\boldsymbol{g}_{\nu}\right)(x, t)-D_{t} s_{\nu}(x, t)+\nu \Delta s_{\nu}(x, t),\right.
\end{array}\right.
$$

where

$$
s_{\nu}(x, t)=K *_{x}\left(\boldsymbol{v}_{\nu} \cdot \nabla \zeta\right)(x, t)=(-1 / 4 \pi) \int_{E_{3}}|x-y|^{-1} \boldsymbol{v}_{\nu}(y, t) \cdot \nabla \zeta(y) d y
$$

and $\boldsymbol{b}(x)=-\nabla s_{\nu}(x, 0)$; for a vector function $\boldsymbol{u}=\left(u_{1}, u_{2}, u_{3}\right)$ we agree that $\Gamma_{\nu} *_{x}, K *_{x} \cdots$ act componentwise and $T_{\nu} * \boldsymbol{u}$ means $\left(\sum_{j=1}^{3} T_{\nu i j} * u_{j}\right)_{1 \leqq i \leqq 3}$. The proof of Theorem 1.1 is thus reduced to the investigation of various terms involved in this formula. Before proceeding we provide some lemmas, most of which are fairly well known.

Lemma 3.1. Let $\Omega, \Omega^{\prime}$ be bounded open sets in $E_{3}$ and let $f(x)$ be in $L^{2}(\Omega) . \quad$ If

$$
u(x)=\int_{\Omega}|x-y|^{-1} f(y) d y,
$$

then $u(x) \in W^{2,2}\left(\Omega^{\prime}\right)$ and the following estimate

$$
\|u\|_{W^{2,2\left(\Omega^{\prime}\right)}} \leqq C\left(\Omega, \Omega^{\prime}\right)\|f\|_{L^{2}(\Omega)}
$$

holds with a constant $C\left(\Omega, \Omega^{\prime}\right)$ depending only on $\Omega, \Omega^{\prime}$.

Lemma 3.2. Let $\Omega$ be an open set in $E_{3}$ and let $f(x)$ be in $L^{2}(\Omega)$. If

$$
u_{\nu}(x, t)=\int_{\Omega} \Gamma_{\nu}(x-y, t) f(y) d y,
$$

then

$$
\left\|u_{\nu}(t)\right\|_{L^{2}\left(E_{3}\right)} \leqq\|f\|_{L^{2}(\Omega)}
$$

for $0<\nu \leqq 1$ and $0<t \leqq T$, and when $\nu \rightarrow 0, u_{\nu}(x, t)$ converges to $f(x)$ in $L^{2}(\Omega)$ uniformly in $t \in(0, T]$. If in addition $f(x) \in C^{\infty}(V)$ in a compact subdomain $V$ of $\Omega$, then as $\nu \rightarrow 0$ every spatial derivative $D_{x}^{\alpha} u_{\nu}(x, t)$ converges to $D_{x}^{\alpha} f(x)$ uniformly for $x \in V$ and $t \in(0, T]$.

Lemma 3.3. Suppose that $\Omega$ is an open set in $E_{3}$ and let

$$
u_{\nu}(x, t)=\int_{0}^{T} \int_{\Omega} \Gamma_{\nu}(x-y, t-\tau) f(y, \tau) d y d \tau
$$

with $f(x, t) \in L^{2}(\Omega \times(0, T))$. Then as $\nu \rightarrow 0, u_{\nu}(x, t)$ converges strongly to the function $\int_{0}^{T} f(x, \tau) d \tau$ in $L^{2}(\Omega \times(0, T))$.

Proof. Write the restriction $\left.u_{\nu}\right|_{Q \times(0, T)}$ as $\Gamma_{\nu, \Omega}(f)$. It is sufficient to show (1) that the operator $\Gamma_{\nu, \Omega}$ is bounded in $L^{2}(\Omega \times(0, T))$ independent 
of $\nu \in(0,1]$, and (2) that as $\nu \rightarrow 0, \Gamma_{\nu, \Omega}(f)(x, t)$ therein converges to $\int_{0}^{t} f(x, \tau) d \tau$ for a dense subset of functions $f$. Now, (1) follows immediately from the estimate in Lemma 3.2. To see (2), we let $f$ be any continuous function on $[0, T]$ with values in $C_{0}^{\infty}(\Omega)$, so that as a function of $x$ the support of $f(x, t)$ is contained in a fixed compact subset $V$ of $\Omega$ for each $t \in[0, T]$. If $\Omega^{\prime}$ denotes any bounded open set in $E_{3}$ including $V$, then by Lemma 3.2, $\int_{\Omega} \Gamma_{\nu}(x-y, t-\tau) f(y, \tau) d y$ converges, as $\nu \rightarrow 0$, to $f(x, \tau)$ uniformly for $x$ in $\Omega^{\prime}$ and for $t$, $\tau$ with $0<\tau<t \leqq T$; on the other hand, the integral converges to zero also uniformly for $x$ in $E_{3} \backslash \Omega^{\prime}$, since we have $\left|\Gamma_{\nu}(x-y, t-\tau)\right| \leqq C \exp (-\gamma / \nu)$ for $0<\nu \leqq 1,(x, y) \in E_{3} \backslash \Omega^{\prime} \times V$ and $0<\tau<t \leqq T$ with constants $C, \gamma$ depending only on $\operatorname{dist}\left(V, \partial \Omega^{\prime}\right)$ and T. From this, clearly follows the convergence of $\Gamma_{\nu, \Omega}(f)(x, t)$ to $\int_{0}^{t} f(x, \tau) d \tau$ in $L^{2}(\Omega \times(0, T))$ for the functions $f$ under consideration, which constitute its dense subspace. Thus we have (2), and therefore the lemma.

LEMMA 3.4. If $\Omega$ is a bounded open set in $E_{3}$, and $h(x, t ; y, \tau)$ is a bounded function for $x, y$ in $\Omega$ and $t, \tau$ in $(0, T)$, then the integral transform

$$
(H f)(x, t)=\int_{0}^{T} \int_{\Omega} h(x, t ; y, \tau)|x-y|^{-\beta}|t-\tau|^{-\alpha} f(y, \tau) d y d \tau
$$

with $\alpha<1, \beta<3$, gives us a completely continuous operator $H$ in $L^{2}(\Omega \times(0, T))$.

Proof. Let $\eta_{\varepsilon}(s)(0<\varepsilon \leqq 1)$ be a function on the reals such that $\eta_{\varepsilon}(s)=1$ for $s \geqq \varepsilon$ and 0 for $s<\varepsilon$ and let

$\left(H_{\varepsilon} f\right)(x, t)=\int_{0}^{T} \int_{\Omega} h(x, t ; y, \tau) \eta_{\varepsilon}(|x-y|) \eta_{\varepsilon}(|t-\tau|)|x-y|^{-\beta}|t-\tau|^{-\alpha} f(y, \tau) d y d \tau$.

The operator $H_{\varepsilon}$ is clearly completely continuous in $L^{2}(\Omega \times(0, T))$; further, a simple computation gives the estimate

$$
\left\|\left(H-H_{\varepsilon}\right) f\right\|_{L^{2}(\Omega \times(0, T))} \leqq C \max \left(\varepsilon^{3-\beta}, \varepsilon^{1-\alpha}\right)\|f\|_{L^{2}(\Omega \times(0, T))}
$$

with a constant $C$ independent of $\varepsilon$, so that as operators in $L^{2}(\Omega \times(0, T))$, we have $H=\lim _{\varepsilon \rightarrow 0} H_{\varepsilon}$. As is well known, the complete continuity of $H$ then follows.

4. Proof of Theorem 1.1. Recall that $\zeta$ is a smooth function such that $\zeta(x)$ is equal to unity on $\Omega_{2 \delta}$ and vanishes identically outside $\Omega_{\delta}$. Thus $\nabla \zeta$ has its support in the closure of $\omega_{\delta}=\Omega_{\delta} \backslash \Omega_{2 \delta}$.

Let us now consider the convergence for $\nu \rightarrow 0$ of various terms of $\left.\boldsymbol{v}_{\nu}\right|_{V \times(0, T)}=\left.\boldsymbol{w}_{\nu}\right|_{V \times(0, T)}$ appearing in (3.3). We first examine 


$$
\nabla s_{\nu}(x, t)=(-1 / 4 \pi) \int_{\omega_{\delta}} \nabla_{x}|x-y|^{-1}\left(\nabla \zeta \cdot v_{\nu}\right)(y, t) d y
$$

Here, by Theorem 2.1, $\boldsymbol{v}_{\nu}$ is in $C\left([0, T] ; L^{2}(\Omega)\right)$ with $\left\|\boldsymbol{v}_{\nu}(t)\right\|_{L^{2}(\Omega)}$ uniformly bounded by a constant for $0<\nu \leqq 1$ and $0 \leqq t \leqq T$, and by Theorem 2.2 $\boldsymbol{v}_{\nu}(t)$ converges weakly in $\boldsymbol{L}^{2}(\Omega)$ as $\nu \rightarrow 0$ for every $t \in[0, T]$. Now the kernel $\nabla_{x}|x-y|^{-1}$ of this integral transform is of class $C^{\infty}$ when $(x, y)$ is in $V \times \omega_{\delta}$. Thus $\nabla s_{\nu}(t)$ converges strongly in $L^{2}(\Omega)$ for each $t \in[0, T]$ and we also have $\left\|\nabla s_{\nu}(t)\right\|_{L^{2}(V)} \leqq C$ with a constant $C$ independent of $\nu \in(0,1]$ and $t \in[0, T]$. By a Lebesgue theorem then follows the strong convergence of $\nabla s_{\nu}$ in $L^{2}(V \times(0, T))$.

The convergence of $\Gamma_{\nu} *_{x}(\zeta \boldsymbol{a}+\boldsymbol{b})(x, t)$ in $\boldsymbol{L}^{2}(V \times(0, T))$ is essentially implied by Lemma 3.2, since $\zeta \boldsymbol{a}$ is in $\boldsymbol{L}^{2}\left(E_{3}\right)$ by assumption and $\boldsymbol{b}(x)=$ $-\nabla s_{\nu}(x, 0)=(1 / 4 \pi) \int_{E_{3}} \nabla_{x}|x-y|^{-1}(\nabla \zeta \cdot \boldsymbol{a})(y) d y$ is also in $\boldsymbol{L}^{2}\left(E_{3}\right)$ by Lemma 3.1 combined with the fact that $\boldsymbol{b}(x)=O\left(1 /|x|^{2}\right)$ for $|x| \rightarrow \infty$.

We now pass on to considering the terms involving Oseen's tensor: $T_{\nu}=\Gamma_{\nu} I+\operatorname{Hess}\left(\Gamma_{\nu} *_{x} K\right)$. Put

$$
\bar{f}(x, t)=(1 / 4 \pi) \nabla \operatorname{div} \int_{\Omega_{\delta}}|x-y|^{-1}(\zeta f)(y, t) d y .
$$

Since $\boldsymbol{f} \in \boldsymbol{L}^{2}(\Omega \times(0, T))$, it follows that $\overline{\boldsymbol{f}} \in \boldsymbol{L}^{2}\left(E_{3} \times(0, T)\right)$ by Lemma 3.1 and by $\overline{\boldsymbol{f}}(x, t)=O\left(1 /|x|^{3}\right)$ for $|x| \rightarrow \infty$. Now, by a change of the order of integration we may express $\operatorname{Hess}\left(\Gamma_{\nu} *_{x} K\right) *(\zeta \boldsymbol{f})$ as $\Gamma_{\nu} * \overline{\boldsymbol{f}}$. Hence,

$$
T_{\nu} * \zeta \boldsymbol{f}(x, t)=\int_{0}^{T} \int_{E_{3}} \Gamma_{\nu}(x-y, t-\tau)(\zeta \boldsymbol{f}+\overline{\boldsymbol{f}})(y, \tau) d y d \tau .
$$

Thus $T_{\nu} * \zeta \boldsymbol{f}$ converges strongly to $\int_{0}^{t}(\zeta \boldsymbol{f}(\tau)+\overrightarrow{\boldsymbol{f}}(\tau)) d \tau$ in $\boldsymbol{L}^{2}(V \times(0, T))$ as $\nu \rightarrow 0$, by Lemma 3.3.

To deal with $T_{\nu} * \boldsymbol{g}_{\nu}$ we first note that the vector $\boldsymbol{g}_{\nu}=-2 \nu \sum_{j=1}^{3} D_{j} \zeta D_{j} \boldsymbol{v}_{\nu}$ $-\nu \Delta \zeta \boldsymbol{v}_{\nu}+p_{\nu} \nabla \zeta$, having support in $\omega_{\delta}$, converges weakly to $p_{0} \nabla \zeta$ in $\boldsymbol{L}^{2}(\Omega \times(0, T))$ for $\nu \rightarrow 0$ by Theorem 2.2. Now take a $C^{\infty}$-function $\eta$ on $E_{3}$ such that $\eta(x)=1$ on $\Omega_{3 \delta}$ and $=0$ outside $\Omega_{2 \delta}$. If $x$ varies in $V$, we may then write:

$$
\begin{aligned}
& T_{\nu} * \boldsymbol{g}_{\nu}(x, t)=\int_{0}^{T} \int_{\omega_{\delta}} \Gamma_{\nu}(x-z, t-\tau) \boldsymbol{g}_{\nu}(z, \tau) d z d \tau \\
& +(1 / 4 \pi) \int_{0}^{T} \int_{\omega_{\delta}}\left(\int_{E_{3} \backslash \Omega_{3 \delta}} \operatorname{Hess}_{x} \Gamma_{\nu}(x-y, t-\tau)(1-\eta(y))\right. \\
& \left.\quad \times|y-z|^{-1} d y\right) \boldsymbol{g}_{\nu}(z, \tau) d z d \tau
\end{aligned}
$$




$$
\begin{aligned}
& +(1 / 4 \pi) \int_{0}^{T} \int_{\omega_{\delta}}\left(\int_{\Omega_{2 \delta}} \Gamma_{\nu}(x-y, t-\tau) \operatorname{Hess}_{y}\left(\eta(y)|y-z|^{-1}\right) d y\right. \\
& \left.-\operatorname{Hess}_{x}|x-z|^{-1}\right) \boldsymbol{g}_{\nu}(z, \tau) d z d \tau \\
& +(1 / 4 \pi) \int_{0}^{T} \int_{\omega_{\delta}} \operatorname{Hess}_{x}|x-z|^{-1} \boldsymbol{g}_{\nu}(z, \tau) d z d \tau \quad\left(=I_{\nu, 1}+I_{\nu, 2}+I_{\nu, 3}+I_{\nu, 4}\right) .
\end{aligned}
$$

Here if $(x, z)$ is in $V \times \omega_{\delta}\left(V \subset \Omega_{4 \delta}\right), 0<\nu \leqq 1$ and $0<t \leqq T$, then we have constants $C, \gamma$, such that $\left|\Gamma_{\nu}(x-z, t)\right| \leqq C \exp \left(-\gamma \delta^{2}\right)$, and also that

$$
\left|\int_{E_{3} \backslash \Omega_{3 \delta}} \operatorname{Hess}_{x} \Gamma_{\nu}(x-y, t-\tau)(1-\eta(y))\right| y-\left.z\right|^{-1} d y \mid \leqq C \exp \left(-\gamma \delta^{2}\right) .
$$

These estimates clearly show that $I_{\nu, 1}(x, t), I_{\nu, 2}(x, t)$ converge to zero in $\boldsymbol{L}^{2}(V \times(0, T))$. Further, by Lemma 3.2 , one sees that $(1 / 4 \pi) \int_{\Omega_{2 \delta}} \Gamma_{\nu}(x-y$, $t-\tau) \operatorname{Hess}_{y}\left(\eta(y)|y-z|^{-1}\right) d y$ converges as $\nu \rightarrow 0$ to $(1 / 4 \pi) \operatorname{Hess}_{x}|x-z|^{-1}$ in $C^{\infty}$-topology, or in particular, uniformly for $(x, z)$ in $V \times \omega_{\delta}$ and for $0 \leqq$ $\tau<t \leqq T$, since $\eta(y)|y-z|^{-1}$ is of class $C^{\infty}$ for $(y, z)$ in $\Omega_{2 \delta} \times \omega_{\dot{\delta}}$. Thus, $I_{\nu, 3}(x, t)$ converges to zero in $\boldsymbol{L}^{2}(V \times(0, T))$. Concerning $I_{\nu, 4}$, it is clear that the integral transform $\boldsymbol{g} \rightarrow \int_{0}^{T} \int_{\omega_{\delta}} \operatorname{Hess}_{x}|x-z|^{-1} \boldsymbol{g}(z) d z$ is a completely continuous mapping from $\boldsymbol{L}^{2}\left(\omega_{\delta} \times(0, T)\right)$ into $\boldsymbol{L}^{2}(V \times(0, T))$. Therefore as $\nu \rightarrow 0 I_{\nu, 4}$ converges strongly in $\boldsymbol{L}^{2}(V \times(0, T))$ since $\boldsymbol{g}_{\nu}$ converges weakly in $\boldsymbol{L}^{2}\left(\omega_{\delta} \times(0, T)\right)$.

As regards the pressure, or its fractional integration $\left.I^{\alpha} p_{\nu}\right|_{V \times(0, T)}=$ $\left.I^{\alpha} q_{\nu}\right|_{V \times(0, T)}$ with $0<\alpha \leqq 1$, if we write $I^{\alpha} q_{\nu}$ as $J_{\nu, 1}+J_{\nu, 2}+J_{\nu, 3}$ in the order corresponding to the three terms in (3.3), then

$$
\begin{aligned}
J_{\nu, 1}(x, t) & =(1 / \Gamma(\alpha)) \int_{0}^{T}(t-\tau)^{\alpha-1} \operatorname{div}\left(K *_{x}\left(\zeta \boldsymbol{f}+\boldsymbol{g}_{\nu}\right)\right)(x, \tau) d \tau \\
& =\int_{0}^{T} \int_{\Omega}|x-y|^{-2}|t-\tau|^{\alpha-1} \boldsymbol{h}(x, t ; y, \tau) \cdot\left(\zeta \boldsymbol{f}+\boldsymbol{g}_{\nu}\right)(y, \tau) d y d \tau
\end{aligned}
$$

where $\boldsymbol{h}(x, t ; y, \tau)$ is a bounded vector function for $(x, t),(y, \tau)$ in $\Omega \times[0, T]$, and by Lemma 3.4, there follows the convergence of $J_{\nu, 1}$ in $L^{2}(V \times(0, T)$ ) as $\nu \rightarrow 0$. The other terms $J_{\nu, 2}, J_{\nu, 3}$ can be treated in a similar way by recalling the weak convergence of $D_{t} \boldsymbol{v}_{\nu}$ in Theorem 2.2 and the uniform boundedness of $\nu^{1 / 2}\left\|\boldsymbol{v}_{\nu}\right\|_{L^{2}\left(0, T ; W^{1,2}(\Omega)\right)}$ in Theorem 2.1. The proof of Theorem 1.1 is thus complete.

5. Remarks. If we suppose in (1.1) the solution $\left(\boldsymbol{v}_{\nu}, p_{\nu}\right)$ to be sufficiently smooth near the boundary, we naturally obtain the so-called compatibility conditions on $\boldsymbol{a}(x), \boldsymbol{f}(x, t)$ and on their derivatives for $x \in \partial \Omega$, depending on the degree of smoothness we require for the solution. For details we refer the reader to [3], and we here assume that such a 
compatibility condition, either in the classical or in a generalized sense, is always satisfied for $\boldsymbol{a}(x), \boldsymbol{f}(x, t)$, according to the situation. In view of regularity results [3], if we assume the data $\boldsymbol{a}, \boldsymbol{f}$ sufficiently smooth, then we may obtain as much regularity as desired for $v_{\nu}, p_{\nu}$; correspondingly, we may state results similar to Theorem 1.1 in a stronger form. We here present such a result without proof, this being analogous to the proof of Theorem 1.1.

THEOREM 5.1. If $\boldsymbol{a} \in \boldsymbol{H}(\Omega) \cap \boldsymbol{W}^{2 k+1,2}(\Omega)$ and $D_{t}^{l} \boldsymbol{f} \in L^{2}\left(0, T ; \boldsymbol{W}^{2 k-2 l, 2}(\Omega)\right)$ for $0 \leqq l \leqq k, k$ being an integer $\geqq 1$, then there exists a unique solution $\left(\boldsymbol{v}_{\nu}, p_{\nu}\right)$ of $(1.1)$ such that $D_{t}^{l+1} \boldsymbol{v}_{\nu} \in L^{2}\left(0, T ; W^{2 k-2 l, 2}(\Omega)\right)$ and $D_{t}^{l} \nabla p_{\nu} \in L^{2}(0, T$; $\left.W^{2 k-2 l, 2}(\Omega)\right)$ for $0 \leqq l \leqq k$. Further, when $\nu \rightarrow 0, D_{t}^{l^{\prime}} \boldsymbol{v}_{\nu}(t)$ converges, uniformly in $t \in[0, T]$, to $D_{t}^{l^{\prime} \boldsymbol{v}_{0}(t)}$ in the space $\boldsymbol{W}^{2 k-2-2 l^{\prime}, 2}(V)$ for $0 \leqq l^{\prime} \leqq k-1$ and $D_{t}^{l^{\prime}} \nabla p_{\nu}(t)$ also converges to $D_{t}^{l^{\prime}} \nabla p_{0}(t)$ in $W^{2 k-2 l^{\prime}, 2}(V)$ for each $t \in[0, T]$, where $V$ is any compact subset of $\Omega$.

\section{REFERENCES}

[1] O. A. Ladyzenskaja, The Mathematical Theory of viscous Incompressible Flow, Gordon and Breach, New York, 1963.

[2] C.W. Oseen, Neuere Methoden und Ergebnisse in der Hydrodynamik, Akademische Verlagsgesellschaft m.b.H., Leiptzig, 1927.

[3] V.A. SolonNIKov, Estimates of the solutions of a nonstationary linearized system of Navier-Stokes equations, Amer. Math. Soc. Translations 75 (1968), 1-116.

[4] R. Temam, Navier-Stokes Equations, North-Holland, Amsterdam, 1979.

DEPARTMENT OF MATHEMATICS

HaChinohe Institute of TeChNology

HACHINOHE, 031

JAPAN 
